\title{
“反思讨论法”在大学有机化学课程教学中的应用探究
}

籍向东 ${ }^{*}$, 曹成

河西学院化学化工学院, 甘肃省河西走廊特色资源利用重点实验室, 甘肃 张掖 734000

摘要: 根据大学有机化学学科特点并结合具体实例, 介绍了 “反思讨论法” 教学的本质、实际意义, 并着重从学生 “学” 的视角和教师 “教” 的视角介绍了 “反思讨论法” 的主要形式, 希望以此助力于充分培养学生的学习主动性、 创造性和练就扎实的基本功, 也期望为大学有机化学的教学探索一种新的教学策略和方法, 获得优质的教学效果。

关键词: 有机化学; 学习反思; 小组式反思讨论法; 主动型

中图分类号: G64; O6

\section{Study of Application of "Reflection and Discussion Method" in the Teaching of College Organic Chemistry}

\section{Xiangdong $\mathrm{Ji}^{*}$, Cheng Cao}

Key Laboratory of Hexi Corridor Resources Utilization of Gansu, College of Chemistry and Chemical Engineering, Hexi University, Zhangye 734000, Gansu Province, China.

Abstract: According to the characteristics of college organic chemistry, this article introduces the essence and practical significance of teaching of the "reflection and discussion method" with examples, particularly focusing on main forms of the "reflection and discussion method" from the perspective of students' "learning" and teachers' "teaching". I hope this will help fully cultivate students' learning initiative, creativity and solid basic skills, and I also hope to explore a new strategy and method for teaching of organic chemistry in universities, to obtain high-quality teaching results.

Key Words: Organic chemistry; Learning reflection; Group reflection and discussion methods; Proactive

有机化学是高等院校化学体系的四大基础课程之一, 是化工化学、生物、药学、医学、农学、 环境、材料等学科的基础课程。在高校中, 开设有机化学课程的目的是通过该课程的学习, 培养学 生具备发现、分析和解决有机化学相关实际问题的能力 ${ }^{[1]}$ 。本文基于笔者讲授的有机化学课程, 提 出了将 “反思讨论法” 应用于教学实践, 对 “反思讨论法” 应用于有机化学课程教学的实际意义进 行思考并介绍其主要实践形式, 期望此教学方法能够调动学生的积极性, 充分发挥他们的学习主观 能动性, 同时也为丰富有机化学授课教师的教学方法提供一个可能的补充视角 ${ }^{[2]}$ 。

\section{1 “反思讨论法” 的本质}

在日常的教学工作中, 教师们苦恼于如何抛出好的问题或是通过精彩的内容讲授以激发学生们

收稿: 2020-05-13; 录用: 2020-06-28; 网络发表: 2020-07-20

“通讯作者, Email: jxdsn@163.com

基金资助: 河西学院教学研究项目(HXXYJY-2017-017) 
参与讨论的积极性, 从而活跃课堂气氛和产生好的学习效果, 但是由这两种途径促发的讨论实际是 一种 “被动型” 的讨论。在有机化学课程的教学中, 可以让学生先对理论学习和实验操作过程中的 问题和经验进行自我反思和总结, 然后在课堂上进一步对反思内容进行讨论, 以加深理解和认知。 笔者将这种教学方法称为 “反思讨论法” , 是学生自主发现问题的 “主动型” 讨论, 其本质是对学 习的经历回过头来再次思考、进行总结或是建立各种认知、想法的连接, 实现合理连接中的建构, 以及无法连接时的质疑。通过学生反思中提到的两个典型常见错误提高认识: 1) 甲苯与苯甲醚的亲 电反应活性判断, 很多学生认为 $\mathrm{O}$ 原子的电负性较大, 这种吸电子作用使苯环电子云密度降低, 从 而判断亲电反应活性: 甲苯>苯甲醚。通过学生的自我反思及教师的指导性提示, 回顾了共轭效应的 概念, 从而明确了 $\mathrm{O}$ 原子不仅有 $-\mathrm{I}$ 效应(吸电子诱导效应), 而且存在 $+\mathrm{C}$ 效应(推电子共轭效应), 且 $+\mathrm{C}$ 效应> $-\mathrm{I}$ 效应, 也就理解了亲电反应活性顺序应为: 苯甲醚>甲苯。2) 醇的脱水反应, 虽然高中 阶段涉及了这一部分知识, 但部分学生仍停留在死记硬背阶段, 在反思中发现自己无法指出在不同 温度下脱水形成烯烃或醚的原因。教师在查看学生反思日记时发现这个问题, 随后在课堂简要提示 了化学键极性的定义等相关概念。学生通过反思了解到, 在一般情况下, 化学键的极性越大, 越容 易断裂。因此分析了形成乙烯需要断裂 $\mathrm{C}-\mathrm{O}$ 和 $\mathrm{C}-\mathrm{H}$ 键, 而形成乙醚需要断裂 $\mathrm{C}-\mathrm{O}$ 和 $\mathrm{O}-\mathrm{H}$ 键, 其中 $\mathrm{O}-\mathrm{H}$ 键的极性大于 $\mathrm{C}-\mathrm{H}$ 键, 因此温度低 $\left(140{ }^{\circ} \mathrm{C}\right)$ 形成乙醚, 温度高 $\left(170{ }^{\circ} \mathrm{C}\right)$ 形成乙烯, 通过 反思将知识串联起来, 增加了对知识的理解性。实现这个本质的关键是教师要转化思维, 破除固有 的想要传授知识、主导过程和关注结果的倾向, 以及等不及学生的反应而主观猜测、判断的行为惯 性。教师除了聆听学生的 “反思讨论”, 还应该研究自己平时的课堂讲授, 深刻反思自己的课堂教 学并与其他教师进行讨论, 教学相长, 通过 “反思-讨论” 的路径, 牢固搭建起学生深入接受知识的 支架。

\section{2 “反思讨论法” 在有机化学教学中的实际意义}

反思是一种有效的学习手段, 学生通过反思自己的学习实践, 明确了今后学习过程中的 “取长 补短” , 既是对已学知识的巩固及升华, 又是对 “知识漏洞” 的及时修复。小组讨论贵在人人参与, 小组中的每个成员在讨论中畅所欲言, 通过短暂的 “头脑风暴” 碰撞了思维的火花, 相互启发, 自 然而然地经历了自主探究的轨迹。“反思讨论法” 的真谛就在于, 不论是教师或学生, 都要明晰自 己的教学(学习)状态, 清楚教学能力(学习能力)的短板及劣势, 敢于怀疑自己, 通过反思后的奋进, 不断迈向新的层次。有机化学的教学不同于一些人文社科的教学, 当教师对教材中化合物定义、物 理性质影响规律及反应机理的推导形成定势思维后, 摆脱不了经验定势、思维定势和教材定势的窠 臼, 甚至陷入了机械重复的教学泥淖之中。“成长=经验 + 反思” 是美国心理学家波斯纳提出的教师 成长公式, 旨在说明: 教师要善于反思, 并在此基础上结合经验积累不断努力提升自己的教学效果, 才能加快自身成长和发展的脚步 ${ }^{[3]}$ 。授课教师开展教学反思并积极讨论, 是直接探究和解决教学中 的实际问题, 不断追求教学实践合理性, 不断提高教学效益和教学科研能力, 促进教师专业化的过 程。在有机化学的教学中, 每个学校面临的问题不尽相同, 工科院校和理科院校在反思方式、反思 内容等方面均有较大区别。

基于自身的教学感受, 工科院校的反思教学应注重与工业生产相互结合, 学生在课后反思中应 着重思考反应条件、催化剂性质等对现实生产的影响。而理科院校的学生应在反思中注重考虑反应 的中间体确定、副产物结构分析、机理推导等方面, 偏重于对有机化学反应中理论问题的提出及解 决。故不同院校学生的培养目标不同, 所面临的问题不同, 则需要反思讨论的重点有所区别, 需要 任课教师根据具体情况应用 “反思讨论法” 提高学生的自我学习能力。因此, 有机化学授课中实行教 师教学反思讨论的意义在于不断地发现困惑, 用 “终身学习” 的自觉, 激活教师的教学智慧, 探索教 材内容的崭新表达方式, 构建师生互动机制及学生学习新方式 ${ }^{[4]}$ 。对于学生而言, 有机化学的特征 是有机物数量庞大、结构复杂、反应产物多、有机物之间可相互转换、电子效应繁杂, 学生普遍反 
映有机化学易懂难记。如乙烯与 $\mathrm{HCl}$ 的加成反应, 从反应表面理解, 仅是 $\mathrm{Cl}$ 原子与 $\mathrm{H}$ 原子分别加 在乙烯中的两个碳原子上, 大多数学生仅记住了这一点。若想真正地理解并记忆, 必须掌握此反应 发生亲电加成反应的机理过程。因此, 若只要求学生被动地接受纷繁复杂的授课信息, 过大的信息 量会造成学生一直保持高度的紧张, 缺少接受知识的基本思考空间, 这样的单边教学会降低学生在 学习中的互动参与性 ${ }^{[5]}$ 。

目前, 有机化学的主要教学方式仍然是课堂教学, 课时少、内容多, 大学教师不能像高中教师那 样细致讲授和透彻阐述, 再加上关注学生知识差异和个性差异的意识薄弱, 授课教师常采用同一种 教学方法对待不同的学生, 这就导致一些基础薄弱的学生跟不上教学进度, 对有机化学的学习产生 了抵触情绪, 学生的学习热情大为降低 ${ }^{[6]}$ 。通过指导学生将有机化学理论知识和实验操作要点相互 结合进行反思讨论, 从而构筑已学知识和新知识相互佐证的思维体系。学生通过对两种知识结构的 反思与讨论, 提高自己对复杂反应机理及人名反应的理解能力 ${ }^{[7]}$ 。现阶段, 大部分高校本科生的学 习任务都很重, 也就出现了部分 “应付上课” 的现象。笔者认为这种 “无思考, 走马观花式上课” 严重阻碍了学生对知识的理解及掌握程度。为了改善这种状况, 第一、将学生对本节课的反思(包括 难理解的内容、疑问等)融入到课后作业中, 教师在批改作业中就可以及时了解学生的学习状态, 对 大部分同学的共性问题可在下一节课进行再次讲解; 第二、小组讨论反思每一章(约 2-3 次课)进行 一次, 时长约 20 分钟, 每个小组长总结小组的主要问题, 然后小组长之间进行时长 5 分钟的问题整 理, 避免了重复问题的出现; 第三、每两章(约 4-5 次课)内容进行一次小组反思汇报, 时长约 30 分钟, 每个小组 10 分钟, 让学生互相分享反思的收获, 在交流中进一步扩展自身的知识面, 发现自身出现 的共性问题, 并进行自我剖析。因此, 一般情况下, 在一学期的课时安排中, 小组反思讨论 25 分钟 $\times$ 5 次 $=125$ 分钟, 小组反思汇报 30 分钟 $\times 4$ 次 $=120$ 分钟, 共计 125 分钟 +120 分钟 $=245$ 分钟 $($ 约 5 学时)。本校的有机化学课时每学期安排为 64 学时, 占用率 $5 / 64=7.81 \%$, 占比率较低, 且此部分 课时也可一定程度上分布于学生的自学课时或答疑课时部分。

综上, 反思讨论在教学中的占用课时相对合理, 且这也是学生所学知识自我升华的必要过程。 此外, “反思讨论法” 还注重学生主体性的发挥, 让其对自己的学习状态真正做到 “心中有数”, 也为教师的教学设计和教学改进提供了有效的实际参考, 有效提高了课堂教学实施效率, 加深了学 生对知识的理解和掌握 ${ }^{[8]}$ 。例如: 笔者在讲授烯烃亲电加成反应时, 会让学生将反思与讨论相结合, 以相辅相成的方式将这部分知识点牢记于心。如图 1 所示, 学生看到反应过程会发现有两种可能性, 一种是生成左侧的仲碳正离子, 一种是生成右侧的伯碳正离子, 但无法确定是哪一种, 且在以后的 考试中会经常容易混淆。因此, 笔者让学生首先反思之前学习的知识点——碳正离子的稳定性, 从 而通过仲碳正离子的稳定性较强得出答案。在反思过程中, 学生以小组讨论形式对电荷密度的分布 情况予以讨论, 左侧仲碳正离子电荷密度 $(0.681)$ 小于右侧伯碳正离子 $(0.732)$, 故可以正确判断 $\mathrm{H}$ 离 子具体进攻丙烯中的哪个碳原子。

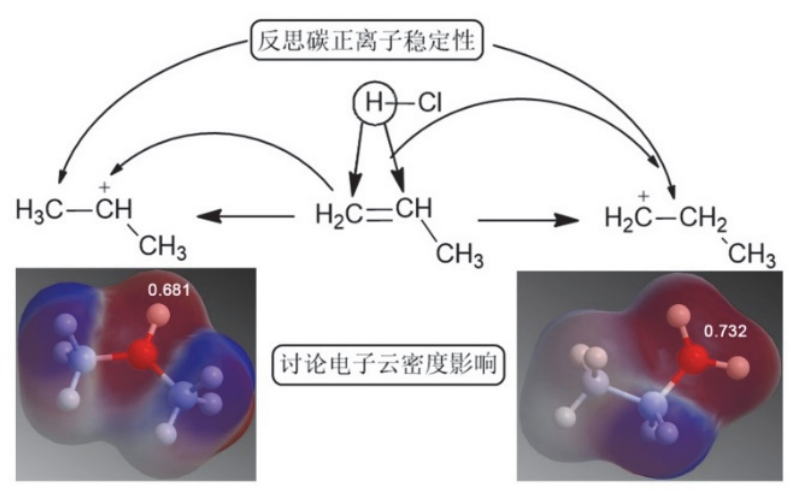

图 1 反思讨论法应用于烯烃亲电加成的反应机理 


\section{3 “反思讨论法” 的主要形式}

\section{1 学生 “学” 的视角: 小组式反思讨论}

“讨论式教学” 是修正传统的 “灌输式教学” 的一种教学策略, 改变了使学生被动接受知识的 学习模式。小组讨论是课堂教学中目标实现的一条途径, 也是学生学习的基本方式之一, 能够激发 学生积极动脑学习的兴趣。有机化学的教学涉及到理论知识和实验操作两部分, 学生在理论知识学 习和实验操作技能掌握的过程中均会遇到疑问和困惑, 甚至理论知识掌握不扎实或者出现断层, 就 会影响实验操作的顺利进行, 出现 “理论不熟悉, 实验不会做” 的恶性循环 ${ }^{[9]}$ 。因此, 在有机化学的 授课过程中, 教师要注意引导学生将反思贯穿于理论学习和实验操作的各个阶段, 进而将反思的结 果进行专门的讨论, 在此过程中教师详细掌握了学生在理论学习和实验操作方面的具体问题和困惑, 以便对症下药; 学生也从学习反思中深刻认识到自己的短板, 通过与其他同学的交流, 既可以解决 一些学习困惑, 又可以受到一些启发, 改善了学习状态 ${ }^{[10]}$ 。比如, 在 “肉桂酸乙酯的制备” 实验中, 学生 $\mathrm{A}$ 的实验产率较其他人低很多, 通过课后的小组反思明确了原因, 主要是由于催化剂用量不足 (浓硫酸一般加入 $1 \mathrm{~mL}$ 左右, 而这位学生仅加入 2 滴) 与无水硫酸镁的用量过多(一般实验课本没有 明确标明用量, 学生加入了约 $5 \mathrm{~g}$ )。课后, 学生 A 将小组反思总结的两大问题记录在了自己的反思 日记中, 通过查阅相关资料并进行自我反思总结, 找到了出现问题的原因。1) 催化剂用量不足, 主 要会造成体系中催化剂浓度偏低, 故产率有所下降, 现已明白催化剂虽然可以循环催化反应, 但也 会有一定的损耗且需要适当的浓度。2) 无水硫酸镁用量过多, 会造成部分产物吸附在无水硫酸镁的 表面, 使产率下降, 现已明白无水硫酸美的使用量一般根据其在溶液中的团聚状态进行判断。学生 A 经过小组反思、自我反思, 找到了实验中的问题, 加深了印象, 并在下次实验前把这些收获分享 给其他同学, 体现了 “反思-分享-反思” 这一良性循环的重要性。

在有机化学教学中采用的 “反思讨论法” , 具体包括理论学习反思讨论和实验操作反思讨论两 部分。在进行反思讨论前, 要按学号把班级同学分成若干小组, 每组 5-6 个人(笔者所在的学校授课 班级人数一般 40 人左右, 分成了 8 组), 每组选出一名小组长, 负责本组讨论的内容记录和结果反 馈。在每次上完理论课后, 教师要求学生在课后对所学内容进行反思, 写好反思日志, 具体内容包 括: 未理解的部分、需要深入了解的部分、课堂学习状态专注程度、实际学习效果等; 在下节课开 始后, 教师给出学生 6-8 分钟时间进行讨论, 让学生对自己的反思结果畅所欲言, 与其他同学进行 相互交流。教师注意时间控制, 讨论结束后, 开始进行新课讲授。各小组组长在课后将讨论内容进 行整理, 交于授课教师, 教师结合学生的反思讨论对教学内容进行查漏补缺和详略得当的调整。

笔者所在学校的有机化学教学是 “理论讲授+实验操作” 模式, 在理论讲授期间根据教学内容穿 插了几次实验操作讲授。这一模式主要是为了增强学生有机化学理论知识与实验操作的关联性认识, 具体是将实验涉及到的理论知识从理论课讲授中转移到实验课讲授中, 最大程度地增加理论知识与 实验的衔接性。比如, “环己烯的制备” 实验, 就会把原为理论课讲授的 “醇的脱水反应” 小节转 移到实验课中进行教授。这一工作现已在进行中, 学院后续将会不断增加这种 “理论讲授+实验操 作” 结合模式的教学形式, 让学生在理解机理的前提下 “深度思考” 地进行实验, 而不是 “头脑空 虚” 地应付了事。因此, 学生同样要对实验操作过程进行小组式的反思讨论, 具体包括: 实验操作 流程是否规范、实验操作是否认真、不会操作部分、已学理论是否能够指导实验操作等。通过对理 论学习和实验操作的小组式反思讨论, 引发学生结合自己的学习实际进行积极思考, 激发学习兴趣, 使他们在学习过程中自觉经历自主探究学习行为的轨迹, 真正学会独立摄取知识的方法, 提高了发现 问题、提出问题、解决问题的能力。此外, 有学生在反思中提出发生在身边的一些现象, 并用化学知 识进行解释, 例如人喝酒为什么会头晕, 就用到了有机化学中醇的氧化知识点, 乙醇在人体内被乙 醇脱氢酶作用氧化为乙醛, 若体内乙醇脱氢酶含量较低, 则无法快速氧化为乙醛, 则会出现醉酒现象。 


\section{2 教师 “教” 的视角: 教学反思讨论的 “三步法”}

\section{2 .1 教学反思日志}

教师在每次有机化学课程教学工作结束后, 要及时对自己的教学进行反思总结, 写出简要的教 学反思日志, 内容大致包括: 凝练升华、教学智慧、学生创新、再教设计等。1) 凝练升华: 即使对 化合物结构及反应机理已理解足够透彻, 在教学的过程中也难免会出现将机理复杂化等现象, 对其 进行及时反思和整理, 有助于扩展教学思路, 提升教学能力。2) 教学智慧: 在机理讲解及讨论的过 程中, 师生偶尔会碰撞出 “灵感的火花”, 基于学生思维的讨论及思考是提升教师教学方法及教学 能力的基石, 故教师课后一定要及时检索回顾这些瞬间并进行记录以提升教学策略。3) 学生创新: 在课堂教学中, 伴随着教师的讲解和引导, 学生会产生一些富有创新性的 “思维火花” , 教师对学 生在课堂上的独特见解给予充分的肯定, 是对学生的一种赞赏和鼓励。这些难能可贵的创新性思维 或见解是对教师课堂教学的一种补充与完善, 教师在课后及时将其记录下来以便应用于今后的教学, 不仅能使学生的好方法、好思路得以推广, 而且可以拓宽教师的教学思路、提高教学水平, 为教学 补充丰富的材料养分。4) 再教设计: 再教设计对于教师的教学实践尤为重要。教师需要在课后静心 沉思, 回忆在课堂教学过程中摸索出了哪些教学规律; 教法上有哪些创新; 组织教学方面有何新 招; 知识点上有什么发现; 解题的诸多误区有无突破; 启迪是否得当; 训练是否到位等等。通过反思, 将这些得失及时记录下来并进行必要的归类与取舍, 写出 “再教设计”, 同时思考再次讲授此部分 内容时应该如何进行。这样可以做到扬长避短、精益求精, 把自己的教学水平提高到一个新的境界 和高度 ${ }^{[11]}$ 。

\subsection{2 课程观摩反思}

有机化学课程观摩反思有两种具体形式。一是授课教师可以相互观摩彼此的教学, 包括理论讲 授课程和实验操作课程, 结合别人的授课方式和授课风格反思自己的教学模式, 取长补短, 提升教 学水平 ${ }^{[12]}$; 二是授课教师可运用录音和录像技术记载自己的理论讲授和实验操作授课过程, 通过课 后回放进行授课反思, 为以后的教学工作提供改进的思路和方向。

\subsection{3 反思结果讨论}

在教研室活动中, 讲授有机化学的不同教师聚在一起, 结合对近段时间以来学生 “学” 的反思 讨论结果和教师 “教” 的反思讨论结果进行充分讨论, 主要是找出问题和总结经验教训。对于教学 过程中的学生 “学” 和教师 “教” 的相关问题, 要共同讨论解决的办法, 得出改善的方案或路径。对于 教学过程中的一些好的经验做法, 教师之间进行充分的交流并相互启发, 同时要注意经验的适用性 问题。对于教师各自在理论课程讲授和实验操作课程讲授的过程中遇到的一些教训, 特别是学生在 实验操作课上发生的一些不可预料情况和突发状况要及时向其他教师分享和提醒, 防微杜渐。此外, 如图 2 所示, 根据上述内容笔者提出了反思讨论法的运行流程, 将反思讨论法分为两个主要部分:

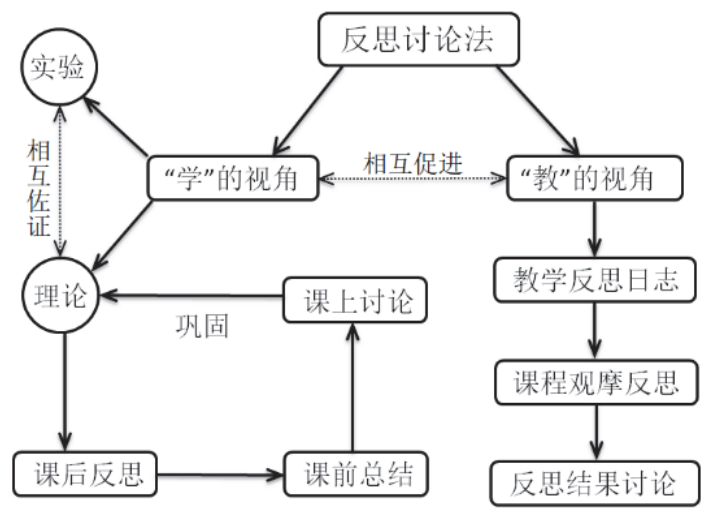

图 2 反思讨论法的运行流程 
学生 “学” 的视角和教师 “教” 的视角。从学生 “学” 的视角, 可以通过反思将有机化学理论知识 点和实验相互佐证, 有利于增加学生对知识点的掌握程度, 提高学习效率。在理论学习部分, 每节 课结束后需要进行课后反思, 经过反思课堂内容和遇到的问题, 在下一节课进行课前总结, 并与小 组成员讨论学习, 以达到巩固理论知识的目的。从教师 “教” 的视角, 教师的视角与学生是相互促 进的关系, 通过教学反思日志、课程观摩反思、反思结果讨论过程提升教学和专业知识水平。上述 的 “两相模式” 可以极大地促进学生学习的主动性和教师的教学能力, 将反思讨论法的理论意义较 好地应用在实践中。

\title{
4 结语
}

在教学过程中使用反思与讨论相结合的 “反思讨论法” , 这种方法应同时适用于学生的 “学” 和教师的 “教” , 在学生学习过程的过程中, 以 “反思讨论法” 为媒介, 能够使师生间的教学相长 变得更加深入和具体。有机化学涉及的基础概念内容庞杂且反应机理抽象难懂, 学生容易出现知识 点混淆和化合物结构理解不深刻, 甚至容易产生排斥厌学心理, 在教学实践当中, 通过课后的学习 反思和课中的小组讨论, 能够帮助学生营造带有问题和目的进行学习的环境, 从而使其内心的学习 兴趣得到充分的挖掘和激发 ${ }^{[13]}$, 促使学生在相互合作中探讨并掌握知识。

在 “反思教学法” 的使用过程中需要注意以下几个关键要点, 以更好地达成优质的学习效果。 一是要关注学生的反思讨论进程并注意掌控时间, 还要特别注意总结归纳讨论的收获和启发。在课 堂讨论正式开始后, 教师要走入学生中间, 倾听他们的学习困惑并通过引用实例、结构模型展示等 形式参与到学生的讨论中去; 对于教研室活动中, 授课教师们之间的讨论也要注意讨论的议程设置, 紧扣主题, 比如有机化学教学的实际困惑、好的课堂教学方法、学生们普遍认为的重难点知识、实 验操作过程中的突发状况的解决方案等。二是可以把学生平时参与 “反思讨论” 的表现纳入有机化 学平时成绩的考核中, 进一步丰富考核评价指标, 既能调动学生的学习积极性, 又能全面、公正地 评价学生的学习情况, 同时也避免了学生抱有 “只要考前突击, 就能通过考试” 的想法, 忽视平时的 知识积累, 考完即忘, 并未牢固掌握有机化学知识。三是教师在有机化学课程教学中注意多引导学 生反思高中与大学化学知识的有效衔接, 教研室活动中的授课教师讨论在议程设置上也要注意将当 前的有机化学的科研前沿与有关教学内容紧密地结合起来, 增强学生对有机化学学科发展方向的了 解和浓厚兴趣, 为今后进入毕业设计或研究生科研工作提早热身。

\section{参 考 文 献}

\author{
[1] 丁锐. 产业与科技论坛, 2017, 16 (15), 228. \\ [2] 王云侠, 白银娟, 李剑利, 赵军龙, 张世平, 魏青, 王兰英. 大学化学, 2020, 35 (1), 23. \\ [3] 张毅敏. 教师, 2013, No. 23, 120. \\ [4] 王正琼. 职业, 2014, No. 11, 162. \\ [5] 俞善辉. 化工高等教育, 2008, No. 3, 83 . \\ [6] 张变香, 郭炜, 冯丽恒. 化工高等教育, 2019, No. 5, 36. \\ [7] 姜林, 万福贤, 李映, 张元红. 高师理科学刊, 2019, 39 (10), 84 . \\ [8] 尹虹. 化学教育, 2020, No. 6, 113 . \\ [9] 李厚金, 赖瑢, 朱可佳, 张利, 陈六平. 大学化学, 2017, 33 (4), 21. \\ [10] 金曦, 江辰. 教育教学论坛, 2017, No. 24, 224. \\ [11] 伍建华, 向延鸿, 刘志雄, 彭晓春. 化工管理, 2019, No. 25, 11. \\ [12] 于青, 王忠卫, 刘蕾, 黄小文. 大学化学, 2018, 33 (2), 18. \\ [13] 蒋雪妹, 许嘉玮, 孙世新, 邢蓉. 广东化工, 2018, 45 (18), 210.
}

